\title{
Fundamentación epistemológica de la vocación policial ${ }^{1}$
}

https://doi.org/10.21830/9789585380202.05

\author{
Álvaro José Estupiñán Henao \\ Elizabeth del Carmen Quinchia Lobo \\ Valentina Quintero Rivera ${ }^{4}$
}

Escuela de Cadetes de Policía "General Francisco de Paula Santander"

Resumen. Este capítulo hace un análisis documental para fundamentar el término vocación policial, uno de los valores más importantes para ejercer la profesión de ser policía. Con este propósito, el estudio emplea una metodología cualitativa, fundamentada en una perspectiva interpretativa. El enfoque se basó en métodos de recolección de datos no estandarizados ni predeterminados completamente. Respecto a los resultados, se identificaron las habilidades socioemocionales/blandas como una de las categorías y subcategorías de estudio. Esto sugiere la construcción del perfil del policía actual, donde se articulan las competencias respecto al saber, saber hacer en cuanto a experiencias adquiridas, y el desarrollo de habilidades que le permitan enfrentar los retos y desafíos de la cotidianidad.

Palabras clave: competencias para la vida; educación militar; formación de investigadores; perfil policial; Policía Nacional de Colombia; sentido de pertenencia; vocación policial.

1 Este capítulo forma parte de los resultados del proyecto de investigación "Educación Policial" del grupo de investigación Ecsan, de la Escuela de Cadetes de Policía "General Francisco de Paula Santander", categorizado en C por Minciencias y con código de registro COL0080809. Los puntos de vista que se presentan en este capítulo pertenecen a los autores y no reflejan necesariamente los de las instituciones participantes.

2 Odontólogo general de la Universidad del Sinú. Estudiante de la Especialización en Servicio de Policía, de la Escuela de Cadetes "Francisco de Paula Santander”, Bogotá, Colombia. Contacto: alvaro. estupian@policia.edu.co

3 Odontólogo general de la Universidad del Sinú. Estudiante de la Especialización en Servicio de Policía, de la Escuela de Cadetes "Francisco de Paula Santander", Bogotá, Colombia. Contacto: ec.quinchia@policia.edu.co

4 Ingeniera industrial de la Universidad Tecnológica de Pereira. Estudiante de la Especialización en Servicio de Policía de la Escuela de Cadetes "Francisco de Paula Santander", Bogotá, Colombia. Contacto: vv.quinter00004@policia.edu.co 


\section{Introducción}

Toda nación debe regirse por una organización que proteja los Derechos Humanos, que es una responsabilidad del Estado. Con este objetivo se crean diferentes organizaciones, entre ellas la Policía, con el propósito de garantizar la seguridad de los ciudadanos y mantener el orden público regido por las órdenes de las autoridades estatales. Teniendo en cuenta lo anterior, el problema de esta investigación parte de la fundamentación del término vocación policial, con la cual se busca que las personas afiancen el sentido de pertenencia al querer ingresar a la institución, motivando la misión, visión y mística policial. La vocación es uno de los valores policiales más importantes para ejercer la profesión de ser policía, según el documento Lineamiento de política de control institucional y veeduría social para el mejoramiento del servicio, que en el segundo capítulo hace referencia a los componentes del proceso de integridad. Específicamente, Naranjo (2010) define este término como "un conjunto de estrategias comunicativas, organizativas y pedagógicas desarrolladas desde el ámbito de la prevención y el control, encaminadas a modelar el comportamiento ético de la mujer y del hombre policía” (p. 22).

Así, cuando se aplican test estándar a los estudiantes que terminan su ciclo de formación de bachillerato para identificar su orientación vocacional (Tintaya, 2016), se puede considerar que estos no son suficientes para definir un aspecto tan importante en el proyecto de vida de cada persona. Según Bulla y Guarín (2015), la vocación policial requiere de

altos estándares de integridad moral, respeto por las normas y la ciudadanía, sensibilidad a los asuntos sociales, alto sentido de pertenencia, que permitan armonizar los proyectos personales con los institucionales, capacidad de controlar las emociones en situaciones de presión y estrés. (p. 10)

De otra parte, en ocasiones se presenta incertidumbre respecto a la vocación policial. Según Lorenz (2018, p. 350), "la muerte es un elemento muy presente en el imaginario policial, sin embargo, no es variable para desistir de ella”, pues es una profesión que ofrece una alternativa de empleo efectiva. En un contexto en el que actualmente cada día son menores las oportunidades que se encuentran en el país para poder ejercer un trabajo digno, la institu- 
ción brinda muchos beneficios, dentro de los cuales se encuentra la estabilidad económica y el derecho a evocar el uso del buen retiro al cumplir los veinticinco años de servicio, beneficio al que ninguna otra institución tiene acceso y que permite a los policías convertirse en sujetos útiles para la sociedad a corto tiempo de edad dedicándose a otras actividades.

Ahora bien, se ha establecido que las razones por las cuales se presentan comportamientos que no están acordes con la disciplina policial son, inicialmente, la inmadurez, la inexperiencia, la falta de vocación, la corta edad de los funcionarios, la falta de responsabilidad, compromiso y desconocimiento de las normas, los cambios generacionales, la falta de exigencia de los comandantes, la pérdida de jerarquía del personal por no superar el concurso para ascender en el escalafón, la falta de motivación y la frustración laboral (Marín, 2018, p. 44).

Esta situación llevó a definir la pregunta de investigación: ¿Cómo fundamentar epistemológicamente la vocación policial buscando la adaptación de las nuevas condiciones sociales y laborales de la sociedad? Según la Resolución 06706 de 2017, "Manual de ciencia, tecnología e investigación de la Policía Nacional de Colombia", se infiere que el área de esta investigación es la "educación" y su línea de investigación es la "formación policial", de manera que con este análisis se contribuye a los procesos educativos. A través del área de investigación quedan explícitos los fundamentos epistemológicos y los enfoques conceptuales de la formación de los policías (art. 26).

Esta investigación tiene como propósito analizar el concepto contemporáneo de vocación policial para consolidar su fundamento en el personal de la institución. Por esta razón, se busca adaptarlo a las nuevas condiciones sociales y laborales de la sociedad, así como enfatizar en su origen epistemológico, doctrinal y subjetivo. Además, se pretende crear líneas de estudio que afiancen la vocación en las instituciones policiales, de tal manera que se forme un ente de policía íntegro y comprometido con la sociedad mediante el fortalecimiento de la doctrina y la mística policial, puesto que estos han sido factores que se han ido perdiendo con el paso del tiempo (Soprano, 2013). Por consiguiente, el estudio analiza, a través de la revisión bibliográfica, la fundamentación epistemológica del constructo, y la conceptualización de la vocación policial, sus categorías y subcategorías. 


\section{Marco teórico}

En este apartado se aborda el desarrollo histórico del concepto de vocación a partir de su noción etimológica y las diferentes concepciones que sobre esta se han generado. Por otro lado, se analizan las principales habilidades socioemocionales de los policías desde la fundamentación etimológica de la vocación policial y su relación con el autocontrol, la identidad, el sentido de pertenencia y la integridad de la persona humana.

\section{Historia de la vocación}

Según Bardales (2017), “etimológicamente, la palabra vocación deriva del latín 'vocare', que significa 'llamar'; de ahí que la vocación, en sentido clásico, era la vocación religiosa, un llamado al sacerdocio, el compromiso con una profesión de fe" (p. 21). Esto indica que, inicialmente, la vocación tenía una fuerte connotación existencial sobre el sentido de la vida del individuo. Para Rodríguez et al. (2017), "el proceso de elección vocacional está conformado por varios elementos, tales como rasgos de personalidad, intereses vocacionales, aptitudes y habilidades" (p. 346) — cabe señalar que la personalidad puede variar dependiendo de cada individuo-.

Asimismo, según Nieto (2019), etimológicamente el concepto de vocación significa "llamada", de manera que este "hace referencia al llamado a satisfacer una necesidad para lograr el bienestar del individuo y afirmar un interés que impulsa a hacer las cosas con gusto y con plena satisfacción; es una combinación de conciencia y voluntad" (p. 154). De acuerdo con Garriga y Maglia (2018), la vocación está "asociada a un fuerte compromiso emocional, a un acto de entrega desinteresado, lo cual suele opacar la condición de trabajador, en donde lo que prima es el intercambio de tareas por un salario" (p. 12).

\section{Habilidades socioemocionales en los policías}

En la actualidad, Colombia afronta una serie de desafíos enmarcados en la construcción de espacios de convivencia que aseguren una paz fuerte y duradera (Fajardo, 2017). En este sentido, la mediación policial se convierte en una herramienta para la prevención y el manejo alternativo de la resolución de los 
conflictos que alteran las relaciones entre el Estado y la sociedad. Asimismo, existe interés por el aprendizaje de competencias y habilidades blandas.

En este contexto, Camacho y Céspedes (2018) consideran "que las características psicosociales asociadas al perfil del policía como mediador son la conciencia, la valoración adecuada, la confianza y la empatía, entendida esta como la capacidad para implicarse en las experiencias emocionales de los demás" (p. 280). Para Muñoz y Madrid (2017), "la importancia de las competencias blandas o habilidades socioemocionales hacen referencia a los conocimientos, las habilidades y las actitudes de una persona que le permiten interactuar con otras de manera efectiva" (p. 45).

\section{Autocontrol}

Es el conjunto de mecanismos psíquicos que intervienen en el proceso de toma de decisiones, más que por una evaluación irracional de las intenciones, por la disposición ambiental sobre la cual actúa la voluntad o por la inadecuación de los medios necesarios para lograr el autocontrol (Landines, 2019, p. 2). Como aprendizaje social es importante destacar tres aspectos relevantes para el estudio del autocontrol: los procesos cognitivos básicos, de internalización, afectivos y motivacionales (Fernández et al., 2010, p. 218).

\section{Sentido de pertinencia}

Se define como el sentimiento de posesión sobre un lugar, espacio u objeto de interés. Por lo tanto, esta emoción es propia del ser humano y es concebida como una necesidad, la cual desarrolla el interés por defender, proteger y cuidar todo lo que le interesa y tiene la capacidad de apropiarse, aun sin ser de su propiedad (García, 2019, p. 6). Finalmente, el sentido de pertenencia y de identidad se refleja en los valores, las costumbres y las manifestaciones culturales que se construyen y mantienen al sentirse parte de una familia, de un grupo o de una nación (Coronado et al., 2016, p. 384).

\section{Integridad}

La integridad tiene una relación estrecha con la autonomía, pero esta descansa en el derecho de todos los seres humanos a su integridad moral como personas, expresada en una relación equilibrada entre los elementos corporales, 
psicosociales e intelectuales. La integridad se refiere a la totalidad del individuo, a los aspectos fundamentales de la vida humana que deben ser respetados (Arruda \& Monteiro, 2017, p. 312).

\section{Métodos}

La metodología que se empleó fue investigación cualitativa, fundamentada en una perspectiva interpretativa. El enfoque se basó en métodos de recolección de datos no estandarizados ni predeterminados completamente (Sampieri et al., 2014, p. 42). Una revisión documentada, a partir de enfoque hermenéutico interpretativo, de textos especializados en las diferentes bases de datos, como son Scopus, Redalyc, Dialnet, Scholar, biblioteca de la Policía Nacional, entre otras.

La técnica de recolección de información consistió en una revisión bibliográfica sistémica. Por su parte, el procedimiento se desarrolló en las siguientes fases:

1. Primera fase. Documentación y elaboración de antecedentes. Se desarrolló el proceso de recolección de datos para el análisis de documentos basándose en la conceptualización de las categorías y las subcategorías, así como en la importancia de la formación policial en autores como Lorenz (2018), Garriga y Maglia (2018), Pérez (2017), Bulla y Guarín (2015), entre otros.

2. Segunda fase. Clasificación de la información. En esta fase se empleó el programa Atlas.Ti para hacer el análisis cualitativo de la información textual.

3. Tercera fase. Análisis e interpretación de la información. Se realizó interpretación de las redes semánticas.

4. Cuarta fase. Discusión y conclusión de la investigación.

\section{Resultados}

El análisis se fundamentó en la recolección de la documentación a través de diferentes bases de datos, registradas en la plataforma Atlas.Ti. Esta docu- 
mentación constituye la fuente documental para interpretar la fundamentación epistemológica del constructo conceptual de la vocación policial al servicio de la sociedad (López, 2019). Específicamente, el análisis se realizó en torno a las siguientes categorías: la primera es el concepto de vocación y las subcategorías vocación policial, de servicio y profesional; la segunda categoría es formación policial y la tercera son las habilidades socioemocionales o blandas, con subcategorías como identidad, integridad, autocontrol y sentido de pertinencia.

A continuación, la figura 1 evidencia que desde la perspectiva de diferentes autores la vocación está asociada, en primera instancia, con un compromiso emocional. Es decir, la vocación debe ser un acto de entrega desinteresado, lo cual suele opacar la condición de trabajador, cuando es vista como una condición de posibilidad, en donde lo que prima es el intercambio de tareas por un salario.

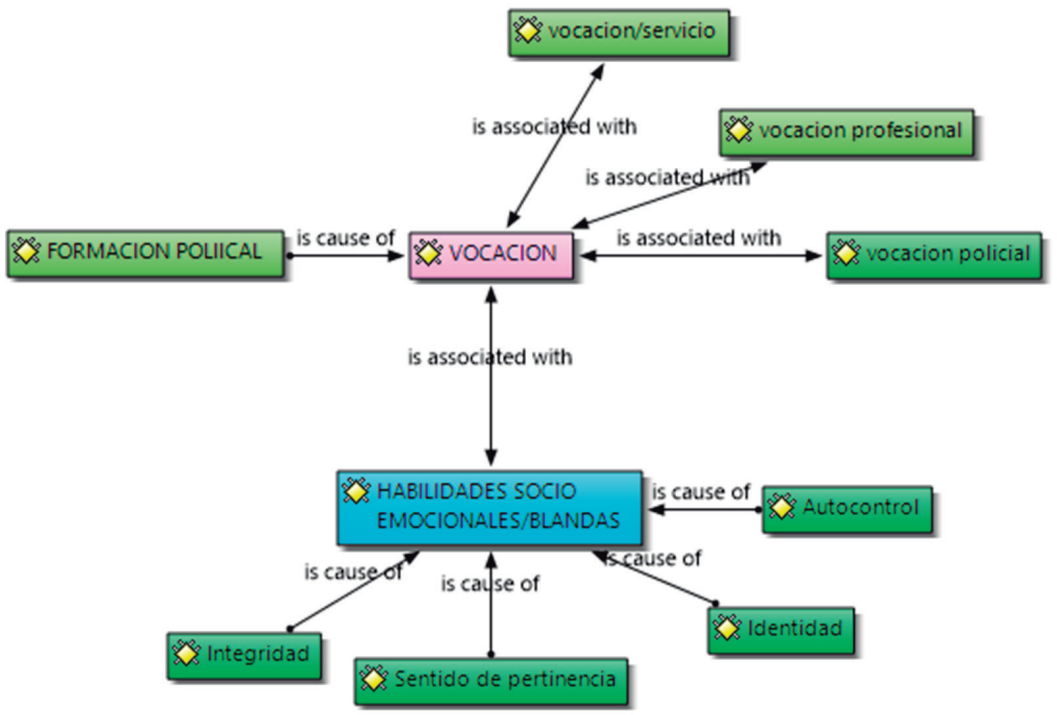

Figura 1. Red semántica de las categorías vocación, formación profesionaly habilidades blandas. Fuente: Elaboración propia.

A partir del análisis de la red semántica, la vocación se comprende como un proceso de elección conformado por determinados elementos, tales como los rasgos de personalidad, los intereses vocacionales, las aptitudes y las habili- 
dades. Sin embargo, esta condición se da dependiendo de cada persona, puesto que no solamente tenemos un rasgo de personalidad, sino que somos personas diversas, tanto en habilidades como en intereses en cada contexto. Dado que en muchos casos existen combinaciones de personalidades, el análisis se realiza de acuerdo con sus aptitudes, características psicológicas y físicas, motivaciones y marcos de referencia socio-económicos y culturales.

En relación con los resultados de la subcategoría vocación de servicio, se puede considerar como un atributo del liderazgo policial, puesto que, a través de la prestación de un servicio profesional y ético, combinado con sabiduría, voluntad y pasión por el desarrollo de sus funciones, refleja una persona satisfecha con su actividad laboral.

De otro lado, la vocación profesional es concebida como un conjunto de acciones de acompańamiento educativo-psicológico social y asesoramiento de manera individual y grupal, que le permite a una persona la toma de decisiones vocacionales y profesionales a partir de concepciones funcionalistas, psicoanalíticas y humanistas. Ahora, desde el punto de vista de la vocación policial, aunque es una tarea riesgosa y requiere de un gran sacrificio, debe verse reflejada en la capacidad de ofrecer un buen servicio a los demás, lo mejor de su condición humana y como funcionario público.

De acuerdo con la Policía Nacional de Colombia (2020), se requiere fortalecer la formación académica policial con un doble propósito: por una parte, implementar una formación integral que le permita afrontar las dinámicas sociales ante la exigencia de la ciudadanía actual, puesto que los policías representan la visión de autoridad, de Estado y de ciudadanos ejemplares, y, por otra, contribuir a la satisfacción de las necesidades de seguridad y convivencia ciudadana. Sumado a lo anterior, se atribuye importancia al liderazgo holístico, el cual, a través de su Modelo Policial Humanístico, busca contribuir al diseño e implementación de buenas y mejores prácticas de liderazgo, que se traducen en mejores condiciones de vida, bienestar y satisfacción. En este orden de ideas, también se busca aplicar un proceso de Modernización y Transformación Institucional por medio de estrategias pedagógicas con énfasis en la formación, basada en competencias profesionales, genéricas y específicas laborales con las dimensiones del ser, saber y hacer. 
Finalmente, las habilidades socioemocionales/blandas se identificaron como una de las categorías y subcategorías de estudio, lo que sugiere la construcción del perfil del policía actual, donde se articulan las competencias respecto al saber, saber hacer en cuanto a experiencias adquiridas, y el desarrollo de habilidades socioemocionales o blandas, entendidas como aquellas aptitudes necesarias para tener un comportamiento adecuado y positivo que permita enfrentar los retos y desafíos de la cotidianidad como autocontrol, identidad, integridad y el sentido de pertinencia, entre otras, y el ser como valores, personalidad y motivaciones.

\section{Discusión}

En relación con el análisis de investigación frente a la conceptualización de la vocación policial, es posible evidenciar que el desempeño, la efectividad policial, se centra en la calidad de la respuesta que ofrece a las demandas ciudadanas en torno a la seguridad y la convivencia. Según Mena (2020), ser policía significa tener una "profesión donde se exige estar siempre al servicio de los demás, un gran compromiso con la sociedad y desempeñar el trabajo con una gran dedicación" (s. p.).

La recopilación de la información permitió evidenciar cómo, desde la perspectiva de autores como Lorenz (2018), el policía tiene una concepción de la muerte y el riesgo como parte de la profesión, pero de igual forma la consideran como una vocación. Algo semejante ocurre desde el punto de vista de Garriga y Maglia (2018), quienes refieren que en la profesión policial el sacrificio y la vocación son valores relevantes.

Paralelamente a esto, García (2019) asevera que el policía sacrifica su tiempo, renunciando a sus intereses personales y relegando su vida privada. De otra parte, para Lorenz (2018), la evidencia más clara de que existe una contradicción entre la vocación y la motivación económica es que, si el elemento económico fue el que determinó el ingreso, no tendrá una carrera duradera en la institución.

Finalmente, los resultados demuestran que la vocación policial implica que, en el contexto de la vida, la toma de decisiones en la profesión policial 
requiere de la capacidad de anteponer cualquier circunstancia laboral, respecto a su vida familiar, lo cual implica darlo todo por la entidad (Garriga, 2016). En consecuencia, es necesario que desarrollen habilidades y destrezas que les faciliten desempeñarse satisfactoriamente dentro del servicio policial.

\section{Conclusiones}

En el análisis se evidenció que los autores tienen una concepción diferente del significado de la vocación, aunque se presenta alguna similitud. Asimismo, coinciden en la importancia que tiene la vocación no solo frente a las diferentes situaciones y escenas que se presentan en el diario vivir de una de las profesiones trascendentales como es la de policía, sino además de tener la vocación de servicio.

Para los autores consultados, la vocación forma parte de la entrega desinteresada que realiza todo individuo en su quehacer, abarca el sentido de pertenencia que se tiene por la institución y por la capacidad de servir a la comunidad sin intercambio alguno, pues esta ha ido interponiendo cambios en el paso del tiempo para encajar en el sistema. No obstante, también se debe aclarar que la vocación también se puede adquirir a través de la experiencia en el ámbito policial.

En cuanto a las habilidades blandas, la bibliografía señala que el autocontrol influye en la toma de decisiones tanto personales como profesionales y vocacionales, de manera que se ha convertido en un punto crítico e indispensable para el desarrollo profesional, teniendo en cuenta variables internas y externas como fundamento para construir su propia identidad.

A lo largo del texto se ha descrito la vocación profesional como una asignatura que debe ser aprendida, a través de un proceso de autoconocimiento de larga duración, ya que en ciertas situaciones el servicio policial no satisface las expectativas laborales y personales de los individuos, pues se ve sesgada por conceptos erróneos e información alterada respecto a las profesiones, ya que cuando las personas ven a un profesional ejercer su labor, no alcanzan a dimensionar las dificultades inherentes a su proceso de formación, sin saber si pueden llegar a superarlas. 


\section{Referencias}

Arruda, D. M., \& Monteiro, S. (2017). Los conceptos de vulnerabilidad humana y la integridad individual para la bioética. Bioética, 25(2), 311-319. https://www.scielo.br/j/bioet/a/ SQkz3G9zHJLvfQPPWntccrK/?format=pdf\&lang=es

Bardales, P. G. (2017). El programa beca 18 como instrumento de igualdad de oportunidades e inclusión social para la realización de la vocación profesional (Tesis de maestría, Universidad Católica del Perú). Repositorio PUCP. http://tesis.pucp.edu. pe/repositorio/bitstream/handle/20.500.12404/9523/BARDALES_PEREYRA_EL_ PROGRAMA_BECA_18_COMO_INSTRUMENTO_DE_IGUALDAD_DE_ OPORTUNIDADES_E_INCLUSION_SOCIAL_PARA_LA_REALIZACION_DE_ LA_VOCACION_PROFESIONAL.pdf? sequence $=1$ \&isAllowed $=\mathrm{y}$

Bulla, P., \& Guarín, S. (2015). Formación policial y seguridad ciudadana: ¿Cómo mejorar el servicio de policia? https://library.fes.de/pdf-files/bueros/kolumbien/11568-20150826.pdf

Camacho, B. E., \& Céspedes, P. N. (2018). Habilidades socioemocionales: Un elemento esencial en el policía como mediador en Colombia. Diversitas: Perspectivas en Psicología, 14(2), 279-295. https://dialnet.unirioja.es/servlet/articulo?codigo=6789736

Coronado, B. A., Moreno L. N., \& Torres, G. A. (2016). Estrategia para el reconocimiento de la identidad cultural y el sentido de pertenencia de inmigrantes latinoamericanos. El Ágora, 16(2), 383-392. https://dialnet.unirioja.es/servlet/articulo? codigo=5622293

Fajardo, E. (2017). Hacia la caracterización de los valores democráticos y ciudadanos de los estudiantes universitarios: Una mirada desde la formación política y la construcción de escenarios de paz. Revista Interamericana de Educación, Pedagogía y Estudios Culturales, 9(1), 87-105. https://revistas.usantotomas.edu.co/index.php/riiep/article/ view/3604/3499

Fernández, S. E., Marín, R. F., \& Urquijo, S. (2010). Relación entre los constructos autocontrol y autoconcepto en niños y jóvenes. Psicología, 16(2), 217-222._https://www.researchgate. net/publication/262647685_Relacion_entre_los_constructos_autocontrol_y_autoconcepto_en_ninos_y_jovenes

García, G. G. (2019). Factores determinantes del sentido de pertenencia en las organizaciones (Trabajo de grado, Universidad Santo Tomás). Repositorio USTA. https://repository.usta. edu.co/bitstream/handle/11634/17423/2019ginagarcia.pdf?sequence $=6 \&$ isAllowed $=y$

Garriga, Z. J. (2016). Los sinsabores del verdadero policía: Representaciones laborales y legitimidad de la violencia policial. Razón y Palabra, 20(2-93), 724-742. https://www.revistarazonypalabra.org/index.php/ryp/article/view/42

Garriga, Z. J., \& Maglia, E. (2018). ¿Qué es un policía? Un estudio sobre las representaciones del trabajo policial. Trabajo y Sociedad, (31), 15-31. https://www.redalyc.org/articulo. oa?id=387359235002

Landines, G. D. (2019). Debilidad de la voluntad y autocontrol: Una discusión en torno a la teoría de las funciones ejecutivas. Interdisciplinaria de Filosofía y Psicología, 14(5), 1-11. https://scielo.conicyt.cl/pdf/limite/v14/0718-1361-limite-14-5.pdf 
López, C. O. (2019). Vocación de servicio en Colombia y nuevas formas de colonialismo en la lógica managerial. Cuadernos de Administración, 32(58). https://doi.org/10.11144/ Javeriana.cao32-58.vscn

Lorenz, M. (2018). Defendiendo la profesión policial. Trabajo y Sociedad, (30), 347-361. https://www.unse.edu.ar/trabajoysociedad/30\%20LORENZ\%20MARIANA\%20 Profesion\%20policial.pdf

Mena, L. C. (2020). La vocación de ser Policía: Razones para ser Policía. https://www.opositor. com/la-vocacion-de-ser-policia/12350

Marín, J. (2018). Actitudes comportamentales con incidencia en la calidad del servicio de Policía. http://168.227.244.104:8080/bitstream/handle/123456789/1401/Informe\%20 final\%20Investigaci\%c3\%b3n\%20Institucional\%202018.pdf?sequence=1 \&isAllowed =y

Muñoz, G. A., \& Madrid, L. E. (2017). Capacitación y competencias blandas como factor de transformación de los recursos humanos: Estrategia mexicana [Ponencia]. XII Congreso Internacional del CLAD sobre la Reforma del Estado y de la Administración Pública, México. http://www2.congreso.gob.pe/sicr/cendocbib/con5_uibd.nsf/ EFA4D8C00A61F4D1052582910081CD61/\$FILE/munozgac.pdf

Naranjo, O. (2010). Lineamiento de politica 7: Control institucional y veeduría social para el mejoramiento del servicio. Imprenta Nacional. http://concursoesagupt. $260 \mathrm{mb}$.net/TOMO\%20 7\%20-\%20Control\%20institucional.pdf?i=1

Nieto A., J. C. (2019). El liderazgo policial: Una herramienta de transformación social. https:// revistalogos.policia.edu.co:8443/index.php/rlct/article/view/551/html

Pérez, M. (2017). "Ser policía es una forma de vida y se necesita vocación de servicio”. Enfoque misiones. http://enfoquemisiones.com/marcelo-perez-ser-policia-es-una-forma-de-viday-se-necesita-vocacion-de-servicio/

Policía Nacional de Colombia. (2020). Talento Humano. Estadísticas de personal. https://www. policia.gov.co/talento-humano/estadistica-personal/cifras

Resolución 06706. (2017). "Manual de ciencia, tecnología e innovación de la Policía Nacional de Colombia”. Grupo de Investigación ECSAN.

Rodríguez, M. M., Sánchez, R. E., \& Labajos, M. M. (2017). Vocación ocupacional y género en estudiantes universitarios de ciencias de la salud. Revista Latinoamericana de Ciencias Sociales, Niñez y Juventud, 15(1), 345-356. http://www.scielo.org.co/pdf/rlcs/v15n1/ v15n1a22.pdf

Sampieri, H. R., Collado, F., C., \& Baptista, L. P. (2014). Metodología de la investigación (6. ${ }^{\text {a }}$ ed.). http://observatorio.epacartagena.gov.co/wp-content/uploads/2017/08/metodologia-de-la-investigacion-sexta-edicion.compressed.pdf

Soprano, G. (2013). Ser militar en la Argentina del siglo XXI: Entre una vocación, una profesión y una ocupación. Revista de Antropología, (23), 71-95. https://www.redalyc.org/ articulo.oa?id=1690/169039923003

Tintaya, C. P. (2016). Orientación profesional y satisfacción vocacional. Revista de Investigación Psicológica, (15), 45-58. http://www.scielo.org.bo/scielo.php?script=sci_ arttext\&pid=S2223-30322016000100004 